\title{
An Analogue Study to Examine the Potency of Group Metacognitive Therapy on OCD Symptoms Among Subclinical OCRD Subjects in Government Head Quarters Hospital at Erode
}

\author{
Sampoornam ${ }^{1}$, Kanaga $^{2}$ \\ 1 Professor, Mental Health Nursing Department, The Tamilnadu Dr.M.G.R. Medical University, Chennai, Dhanvantri College of Nursing \\ Pallakkapalayam, Namakkal, Tamilnadu, India \\ $2 \mathrm{MSc}(\mathrm{N})$ II Year, Dhanvantri College of Nursing Pallakkapalayam, Namakkal, Tamilnadu, India \\ *Corresponding Author: Dr. Sampoornam, Professor, Mental Health Nursing Department, The Tamilnadu Dr.M.G.R. Medical \\ University, Chennai, Dhanvantri College of Nursing Pallakkapalayam, Namakkal, Tamilnadu, India. \\ Email: sampoornamwebster@yahoo.in
}

Received: October 17, 2020; Accepted: January 22, 2021

\section{Abstract}

Context: The analogue study involves subjects that are analogous or similar to the actual clinical phenomena of interest. In this study subjects with very mild symptoms of OCD were tested. Aim: The present study aimed to examine and compare the potency of Group Metacognitive Therapy on OCD Symptoms among Subclinical OCRD Subjects between experimental and control group. Methods: An analogue experimental design was used in this study. Simple random sampling technique was used for selecting 50 samples, in which 25 samples in experimental group and 25 in control group. The tools used for the study were background variables and Yale-brown Obsessive-Compulsive Scale (Y-Boc). Group Metacognitive Therapy was intervened for 4 weeks, with duration of 30-45 minutes. Findings: Results revealed that Paired 't' test value within the experimental group (pretest and posttest) showed statistical significance $(t=9.85)$ among subclinical OCRD subjects. Paired $t$ test value within the control group (pretest and posttest) showed not quite statistical significance $(t=2.00)$ among subclinical OCRD subjects. Unpaired t test value (between experimental and control group) showed statistical significance $(t=8.23)$. Based on posttest OCD symptoms mean scores (Mean=5.24), experimental group showed reduced OCD symptoms compared with control group posttest OCD symptoms mean scores (Mean=7.96). The study findings revealed that administration of group metacognitive therapy were significant in reducing OCD symptoms among subclinical OCRD subjects. This analogue study had statistically proved the significant potency of Group Metacognitive Therapy by curtailing and scaling down the very mild symptoms of OCD.

Keywords: Analogue Study, Group Metacognitive Therapy, OCD, Subclinical OCD.

\section{INTRODUCTION}

Literature on the prevalence and clinical correlates of OCD and subthreshold OCD in Indian population is sparse. There are scarce data on the prevalence of adult obsessive-compulsive disorder (OCD) in India. Lifetime prevalence of OCD in India is $0.6 \%$. It causes distress and interference in functioning. Males have earlier age of onset and are more likely to be unmarried. Early onset was associated typical presentation like presence of sexual obsessions, hoarding repeating rituals and need to touch compulsions. Cleaners and washers were mostly females. Dirt and contamination was the most common content of obsession [13].
Subclinical OCD is defined as the existence of repeated thoughts and actions without functional impairment. The research findings neither suggest that subclinical OCD possess the neutral ground between adult OCD and neither having nor yet condition in terms of obsessive-compulsive symptoms, tics and associated mood/anxiety disorders ${ }^{[4]}$.

The analyses reported that subjects with subclinical OCD symptoms had poorer cognitive processes, lower achievement and lower intelligence-achievement correlation than the healthy matched controls. Findings signify the need for detection of subclinical obsessive-compulsive symptoms and early 
intervention [15]. Subclinical OCD symptoms are relatively common in nonclinical populations and share common neurobiological substrates with clinical OCD [14].

Few studies have tested comorbid patterns in subthreshold OCD subjects and found to be associated with elevated odds for substance abuse, mood, anxiety disorders and somatoform disorders when compared to the non-OCD group. Subjects had lower rates of comorbidity in comparison of OCD subjects with matched controls [1].

Subclinical obsessions and compulsions should be taken into account in research. Symptoms occur not only in obsessivecompulsive disorders (OCD) but are also found in general populations with subclinical obsessive-compulsive (SOC) symptoms, which are considered to be traits and symptoms of OCD that are not severe enough to meet OCD criteria ${ }^{[24]}$.

Metacognitive Therapy (MCT) aimed at teaching clients about metacognitive mode and also integrates cognitive strategies and behavioural experiments, aimed at modifying maladaptive metacognitive beliefs in lieu content of anxious beliefs themselves. Metacognitive treatment appears to be particularly well suited to a group approach. Based on the metacognitive model of OCD [20] therapy focuses on modifying patients 'thoughts about thoughts'. Patients are encouraged to challenge their beliefs about the importance and power of thoughts. The focus is on altering the patient's relationship with their thoughts as opposed to challenging the actual content of the thought.

Metacognitive Therapy has been effective in reducing symptoms in patients suffering from pure obsessions with covert compulsions. The results indicated that Metacognitive Therapy is effective in reducing obsessive compulsive symptoms and in modifying metacognitive beliefs and thought-fusion beliefs [23].

Analogue samples are oftentimes used to study obsessivecompulsive (OC) symptoms and the related facts. This approach was based on the hypothetical testing that result in samples that are relevant to understand $O C D$ symptoms in individuals diagnosed with obsessive-compulsive disorder (OCD). The available evidence suggests research with analogue samples is highly relevant for understanding OC symptoms. Guidelines for the appropriate use of analogue designs and samples are suggested. Researchers use analogue samples to study OCrelated phenomena based on the assumption that the results derived from such samples are relevant and generalizable to understanding these phenomena in individuals with a diagnosis of OCD [9].

Dearth of nursing research literature on analogue study in metacognitive therapy for OCD Symptoms initiated the investigator to conduct this study. The novelty of this research work surged on Group Metacognitive Therapy for OCD Symptoms among Subclinical OCRD subjects. This study focused on subjects with very mild symptoms of OCD, henceforth analogue design was undertaken to draw generalization.

\section{METHODS}

This study was approved by Institutional Human Ethical Committee. After obtaining written informed assent/consent, subclinical OCRD subjects who fulfill the inclusion criteria such as with age between 20 and 60, both gender, very mild symptoms of OCD\& with comorbid psychiatric conditions were recruited and enrolled in the study between February and March
2020. The total sample size was 50, 25 in experimental group and 25 in control group. Simple random technique was adopted in this study. Sampling frame was made, consecutively list of elements numbered and by adopting random number table samples were selected in experimental and control group. Analogue samples with very mild symptoms of OCD were recruited in this study.

Subclinical OCRD subjects with comorbid psychiatric conditions under intellectual insight and true emotional insight (Clinical Rating of Insight -6 point scale) were selected in experimental and control group. Participants were randomly allocated by using random number table either to experimental group or control group.

All participants were interviewed before intervention by assessing background variables likeage, gender, education, occupation, comorbid psychiatric conditionsand blood group. The levels of OCD Symptoms were assessed by Yale-brown Obsessive -Compulsive Scale (Y-Bocs). Immediately after pretest the participants received Group Metacognitive Therapy which comprises of group sessions. The sessions schedule were as follows

Session I-Attention Training Techniques (ATT)

Session II- Identification, rumination \& Group assignments

Session III-Detached mindfulness (DM) \& Group assignments

Session IV- Challenging negative metacognitive beliefs.

Session I was administered during the first week, Session II was administered during second week, Session III was administered during third week and Session IV was administered during fourth week. Group Metacognitive Therapy was intervened for 4 weeks, with duration of 30-45 minutes. Control group received standard methods of care. Posttest was conducted by using the same assessment techniques for all participants. Pilot study was executed to check the feasibility and time requirement of the study. Risk benefit ratio was calculated. Confidentiality of the data was ensured throughout the study.

Descriptive, parametric and nonparametric statistical methods were used to analyze and interpret the data. The data were expressed as frequency, percentage distribution, mean, SE. Paired ' $t$ ' test \& Unpaired ' $t$ ' test was used for estimating the potency of group metacognitive therapy in experimental and control group. Chi square test was used to associate the OCD symptoms with selected background variables. A probability of 0.05 or less was taken as statistically significant. Statistical package for social science, PCT version 17(SPSS Inc, Chicago) was used for analyzing the data.

\section{RESULTS}

Distribution of background variable in experimental group according to their age group depicts that highest percentage $(52 \%)$ were in the age group of 41-60 years. In control group according to their age group highest percentage (56\%) were in the age group of $41-60$ years. The least percentage (48\% and $44 \%$ ) of the subjects were in the age group between $20-40$ years in experimental and control group respectively.

In experimental group gender delineates that highest percentage $(56 \%)$ were female, likewise in control group gender portrays highest percentage $(60 \%)$ were in female. The least 
percentage (44\% and $40 \%$ ) of the subjects were male in experimental and control group respectively.

Education revealed that highest percentage (44\%) was graduate in experimental group and in control group majority (80\%) were schooling. However (36\%) had no formal education and the least $(20 \%)$ had schooling in experimental group, although $(12 \%)$ were graduates in control group and the least $(8 \%)$ had no formal education in control group.

Distribution of background variable in experimental group according to their occupation reveals highest percentage $(60 \%)$ was employed respectively. In control group majority percentages $(52 \%)$ were unemployed. The least $(40 \%)$ were unemployed in experimental group and (48\%) were employed in control group.

Distribution of background variable in experimental group according to their comorbid psychiatric conditions delineates that highest percentage (40\%) had anxiety disorder, likewise in control group majority (40\%) had depression. However (36\%) had psychosis and the least (24\%) had depression in experimental group, although (32\%) had psychosis in control group and the least (28\%) had anxiety disorder in control group.

Blood group showed that highest percentages (40\%) wereA Blood Group in experimental group. In control group highest percentage $(32 \%)$ wereA and B Blood group respectively. Whereas $32 \%$ were OBlood group in experimental group, besides 20\% were BBlood group in experimental group and $24 \%$ were OBlood group in control group. However least $(8 \%$ and
$12 \%$ ) were $A B$ Blood group in experimental and control group respectively.

In pretest, experimental group showed $0 \%$ with no symptoms of OCD and $100 \%$ of the subjects had very mild symptoms of OCD. In posttest, $20 \%$ had no symptoms of OCD and $80 \%$ of subjects showed very mild symptoms of OCD.

In pretest, control group showed $0 \%$ with no symptoms of OCD and $100 \%$ of the subjects had very mild symptoms of OCD. In posttest, $4 \%$ had no symptoms of OCD and $96 \%$ of the subjects had very mild symptoms of OCD.

Comparison of the mean of pretest OCD symptoms scores in experimental group showed 7.04 and posttest showed 5.24. Likewise, comparison of the mean of pretest OCD symptoms scores in control group showed 8.16and posttest showed7.96 (Table 1).

Paired t test value within the experimental group (pretest and posttest) showed statistical significance ( $t=9.85)$ among subclinical OCRD subjects. Paired t test value within the control group (pretest and posttest) showed not quite statistical significance $(t=2.00)$ among subclinical OCRD subjects. Unpaired t test value (between experimental and control group) showed statistical significance $(t=8.23)$. It indicates that there was statistically significant difference between the groups. Based on posttest OCD symptoms mean scores Mean=5.24), experimental group showed reduced OCD symptoms compared with control group posttest OCD symptoms mean scores (Mean=7.96) (Table 1).

Table 1: Comparison of mean, Paired 't' test \& Unpaired 't' test value with pre and posttest scores of OCD symptoms between experimental and control group.

\begin{tabular}{|c|c|c|c|c|c|c|}
\hline \multirow[t]{2}{*}{ Variable } & \multirow[t]{2}{*}{ Group } & \multirow[t]{2}{*}{ Mean, SE } & \multicolumn{2}{|c|}{ Significance paired ' $t$ ' test } & \multicolumn{2}{|c|}{ Significance unpaired 't' test } \\
\hline & & & $\begin{array}{l}\text { Experimental group } \\
\text { pre-posttest }\end{array}$ & $\begin{array}{l}\text { Control group } \\
\text { pre-post test }\end{array}$ & $\begin{array}{l}\text { Experimental } \\
\text { group posttest }\end{array}$ & $\begin{array}{l}\text { Control group } \\
\text { posttest }\end{array}$ \\
\hline \multirow[t]{4}{*}{ Back ground } & Experimental pretest & $7.04,0.31$ & \multirow{4}{*}{$\begin{array}{l}t=9.85 \\
P<0.0001\end{array}$} & \multirow{4}{*}{$\begin{array}{l}t=2.00 \\
P>0.05\end{array}$} & Mean $=5.24$ & Mean $=7.96$ \\
\hline & Experimental posttest & $5.24,0.25$ & & & \multirow{3}{*}{\multicolumn{2}{|c|}{$\begin{array}{l}t=8.23 \\
P<0.0001\end{array}$}} \\
\hline & Control pretest & $8.16,0.24$ & & & & \\
\hline & Control posttest & $7.96,0.22$ & & & & \\
\hline
\end{tabular}

Significant association was obtained between posttest OCD symptoms scores and gender \& comorbid psychiatric conditions $\left(X^{2}=4.16\right.$ and $X^{2}=8.50$; Significant) respectively. Whereas no significant association was observed between posttest OCD symptoms scores for age, education, occupation and blood group in experimental group. No significant association was observed between post-test OCD symptoms scores for age, gender, education, occupation, comorbid psychiatric conditions and blood group in control group.

\section{DISCUSSION}

Hagen K, 2017 conducted an analogue study on metacognition and obsessive-compulsive symptoms in psychosis. Manifold studies have depicted that obsessive-compulsive disorder (OCD) is a common comorbidity in patients with psychotic disorders. Still there are scarce knowledge about the relationship between symptoms of OCD and psychosis. Findings indicate that obsessive beliefs, severity total OCD and metacognitive beliefs were associated with total quality of life scores. Findings indicate that the metacognitive beliefs associated with OCD symptom severity are different from that associated with quality of life.

Laura E. Fabricant, 2015 examined the relationship between analogue and clinical samples in order to better understand the generalizability of data from the analogue samples. Specifically, this study compared an OC analogue sample to an OCD clinical sample, as well as to a healthy control group.

Finally, an important area of difference between sub-clinical and clinical OCD may be in the relationship between obsessional beliefs and OC symptoms. Taken together these results provide general support for the use and utility of $\mathrm{OC}$ analogue samples 
and are largely consistent with dimensional perspectives of OCD.

Analogue samples are often used to study obsessivecompulsive (OC) symptoms and related phenomena. This approach is based on the hypothesis that results derived from such samples are relevant to understanding $O C$ symptoms in individuals with a diagnosis of obsessive-compulsive disorder (OCD). The purpose of this review was to synthesize various lines of research examining the assumptions of using analogue samples to draw inferences about people with OCD. Considering the relevance of analogue samples in OCD treatment executed in this research. The available evidence suggests research with analogue samples is highly relevant for understanding OC symptoms. Guidelines for the appropriate use of analogue designs and samples are suggested [9].

Brasilia, 2020 investigated the application of nursing outcomes and indicators tabbed from the Nursing Outcomes Classification (NOC) to figure out patients with obsessive-compulsive disorder (OCD) in outpatient follow-up. The instrument was tested on patients with OCD undergoing Group Cognitive Behavioral Therapy (GCBT). Four NOC outcomes and 17 indicators were selected. There was a significant change in the scores of nine indicators. The study showed feasibility for evaluating symptoms of patients with OCD through NOC outcomes and indicators in an outpatient situation.

The potency of Group Metacognitive Therapy on OCD Symptoms among Subclinical OCRD Subjects was not widely discussed in many electronic data base including journals, grey literatures and books in the field of nursing. This topic is of great concern because the testing of hypotheses proved reduction in OCD symptoms after the implementation of group metacognitive therapy among subclinical OCRD subjects. The novelty of this research work concern on efficacy of group metacognitive therapy, as one of the most effective nursing intervention employed by the nursing personal to reduce the level of OCD symptoms.

\section{CONCLUSION}

The potency of Group Metacognitive Therapy on OCD Symptoms among Subclinical OCRD Subjects was not widely discussed in many electronic data base including journals, grey literatures and books in the field of nursing. This topic is of great concern because the testing of hypotheses proved reduction in OCD symptoms after the implementation of group metacognitive therapy among subclinical OCRD subjects. The novelty of this research work concern on efficacy of group metacognitive therapy, as one of the most effective nursing intervention employed by the nursing personal to reduce the level of OCD symptoms.

Based on the findings of the study the following conclusions were drawn. The study findings revealed that administration of group metacognitive therapy were significant in reducing OCD symptoms among subclinical OCRD subjects. This analogue study had statistically proved the significant potency of Group Metacognitive Therapy by curtailing and scaling down the very mild symptoms of OCD. Nurses can intervene group metacognitive therapy in clinical settings while handling subclinical OCRD subjects.

Group Metacognitive Therapy can be implemented to reduce OCD symptoms among subclinical OCRD subjects as an integral function of the psychiatric nurse in hospital and community settings. Group Metacognitive Therapy can be added and revised in the nursing curriculum. Nurse administrator can organize an outreach awareness program for psychiatric nurses working in hospital and community setting for improving the competency in the implementation of Group Metacognitive Therapy among subclinical OCRD subjects. Evidence based clinical practice guidelines can be incorporated in Group Metacognitive Therapy as an effective treatment plan strategy to reduce the level of OCD symptoms among subclinical OCRD subjects.

\section{Recommendations}

1. This study can be done for extended duration with follow up in order to observe intensity in reduction of OCD symptoms among subclinical OCRD subjects.

2. This study can be further replicated with other comorbid psychiatric conditions.

3. This study can be done further with moderate to severe levels of OCD symptoms.

4. This study can be conducted further among clinically diagnosed OCRD patients.

5. This study can be done further by executing comparison between Group Metacognitive Therapy and other psychotherapies for subclinical OCRD subjects.

6. This similar study can be replicated on large sample size and there by findings can be generalized for large population.

\section{REFERENCES}

1. Adam Y, Meinlschmidt G, Gloster AT, Lieb R. Obsessivecompulsive disorder in the community: 12-month prevalence, comorbidity and impairment. Soc Psychiatry PsychiatrEpidemiol 2012; 47:339-49.

2. Arlington VA. American Psychiatric Association. Diagnostic and statistical manual of mental disorders. 2000; 4th ed.

3. Costas Papageorgiou. Group Cognitive-Behavior Therapy or Group Metacognitive Therapy for Obsessive-Compulsive Disorder? Benchmarking and Comparative Effectiveness in a Routine Clinical Service. Front. Psychol. December 2018.

4. Donald W Black, Gary R Gaffney. Subclinical obsessivecompulsive disorder in children and adolescents: additional results from a "high-risk" study. CNS Spectr. 2008; 13(9 Suppl 14):54-61.

5. Fineberg NA, Hengartner MP, Bergbaum C, Gale T, Rössler W, Angst J. Lifetime comorbidity of obsessive-compulsive disorder and sub-threshold obsessive-compulsive symptomatology in the community: Impact, prevalence, socio-demographic and clinical characteristics. Int J Psychiatry ClinPract 2013; 17:188-96.

6. Fisher PL, Wells A. Metacognitive therapy for obsessivecompulsive disorder: A case series. J BehavTherExp Psychiatry. 2008; 39:117-32.

7. Franziska Miegel. Metacognitive Training for ObsessiveCompulsive Disorder: a study protocol for a randomized controlled trial. BMC Psychiatry, 2020; 20:350.

8. Janardhan Reddy YC. An overview of Indian research in obsessive compulsive disorder. Indian J Psychiatry. 2010; 52(Suppl1): S200S209.

9. Jonathan S. Abramowitz. The relevance of analogue studies for understanding obsessions and compulsions. Clinical Psychology Review 34 (2014) 206-217.

10. Judith Gellatly. Psychological interventions in obsessive compulsive disorder. Nursing Standard, 2014.

11. Kim Melchior. Metacognitive therapy versus exposure and response prevention for obsessive-compulsive disorder: study protocol for a randomized controlled trial. BMC, 2019. 
12. Laura E. Fabricant. The Utility of Obsessive Compulsive Analogue Research: A Comparison of OCD Patient, Analogue, and Nonclinical Samples. A dissertation submitted in the Department of Psychology (Clinical Psychology), 2015

13. Manjunath Rajashekharaiah \& PravinVerma. Phenomenology of Obsessions and Compulsions in Indian Patients. International Journal of Contemporary Medical Research. 2016; 3(7):2139-2143.

14. Marcello Spinella. Mood in relation to subclinical obsessivecompulsive symptoms. International Journal of Neuroscience, 2005; 115(4):433-43.

15. Partha Malakar, Jayanti Basu. Subclinical obsessive-compulsive symptoms, cognitive processes, school achievement, and intelligence-achievement relationship in adolescents. International Journal of School \& Educational Psychology. 2017; 5(2):115-125.

16. Rees CS, Anderson RA. A review of metacognition in psychological models of obsessive-compulsive disorder. ClinPsychol 2013; 17:18.

17. Samantha Fitt and Clare Rees. Metacognitive Therapy for Obsessive Compulsive Disorder By Videoconference: A Preliminary Study. Behaviour Change. 2012; 29(4):213-229.

18. Subramaniam M, Abdin E, Vaingankar JA, Chong SA. Obsessive Compulsive disorder: Prevalence, correlates, help-seeking and quality of life in a multiracial Asian population. Soc Psychiatry PsychiatrEpidemiol 2012; 47:2035-43.

19. Sunderarajan Padmanabhan. Indian scientists identify cells linked to obsessive-compulsive behaviour. Science \& Technology, 2018.

20. Wells,A.(2000).Emotionaldisordersandmetacognitions:Innovativec ognitivetherapy.WestSussex,UK:JohnWiley\&Sons.

21. World Health Organisation. Projections of Mortality and Burden of Disease, 2004-2030. [Last accessed on 2016 Jul 26].

22. Yoshinaga et al., Development of Nursing Guidelines for Inpatients with Obsessive-Compulsive Disorder in Line with the Progress of Cognitive Behavioral Therapy: A Practical Report. J Depress Anxiety. 2014; 3:2.

23. Zahra Andouz. The Efficacy of Metacognitive Therapy on Patients Suffering from Pure Obsession. Iran J Psychiatry. 2012 Winter; $7(1): 11-21$.

24. Zhu C, Yu F, Ye R, Chen X, Dong Y, Li D, et al. External Error Monitoring in Subclinical Obsessive-Compulsive Subjects: Electrophysiological Evidence from a Gambling Task. PLoS ONE 2014; 9(3): e90874. 